\title{
Morphology, differentiation and adhesion molecule expression changes of bone marrow mesenchymal stem cells from acute myeloid leukemia patients
}

\author{
QIULEI CHEN, YAN YUAN and TONG CHEN
}

Department of Hematology, Huashan Hospital, Fudan University, Shanghai 200040, P.R. China

Received May 29, 2013; Accepted November 4, 2013

DOI: $10.3892 / \mathrm{mmr} .2013 .1789$

\begin{abstract}
Bone marrow mesenchymal stem cells (BMSCs) have been identified as an important component of the hematopoietic stem cell (HSC) niche, which is essential for the maintenance of HSCs. HSC niche alternation has been considered to be the main cause of acute myeloid leukemia (AML). However, little is known with regard to BMSC alteration in AML patients. BMSCs were collected from 10 AML patients and 13 controls in order to examine the morphology, differentiation and adhesion molecule expression changes. It was observed that primary BMSCs from AML patients exhibited aberrant morphologies compared with those from the controls. Prior to adipogenic differentiation, the mRNA and protein levels of the lipid marker gene lipoprotein lipase, from the BMSCs of AML patients, were significantly higher. lipid drops were present early during differentiation in the BMSCs of AML patients and exhibited greater numbers later. Following adipogenic differentiation, the mRNA level of E-cadherin in the BMSCs of AML patients was significantly lower than that identified in the BMSCs of the control groups. Following osteogenic induction, the mRNA level of E-cadherin in the BMSCs of AML patients was significantly higher than in the controls. Therefore BMSCs from the AML patients exhibited irregular morphology, tendency to pre-differentiate to adipocytes and different adhesion molecule expression following differentiation. These differences may further our understanding of the HSC niche in the pathological condition.
\end{abstract}

Correspondence to: Dr Tong Chen, Department of Hematology, Huashan Hospital, Fudan University, 12 Wulumuqi Middle Road, Shanghai 200040, P.R. China

E-mail: chentong@fudan.edu.cn

Key words: acute myeloid leukemia, bone marrow mesenchymal stem cells, hematopoietic stem cell niche, adipogenic, osteogenic, adhesion molecule

\section{Introduction}

Bone marrow mesenchymal stem cells (BMSCs) are self-renewable stem cells that may be differentiated into various cell types in vitro (1). BMSCs have also been identified as an essential component in the hematopoietic stem cell (HSC) niche, as in vivo BMSC depletion reduces HSC content (2). The majority of the HSC niche is comprised of BMSCs, osteoblasts, adipocytes, vascular endothelial cells and the extracellular matrix (3). The HSC niche is essential for the maintenance of HSCs (4). Following niche disruption, HSCs may proliferate and differentiate abnormally.

Acute myeloid leukemia (AML) is the most common type of malignant myeloid disorder in adults and there is evidence to suggest that, apart from gene mutations in HSCs, alterations in the HSC niche have an important role in the proliferation, differentiation and migration of malignant stem cells (5). However, little is known with regard to the alteration of BMSCs in the HSC niche of AML patients, which limit the understanding of AML pathology.

In this study, BMSCs were isolated from AML patients and controls and their morphologies, differentiation capabilities and adhesion molecule expression, which were hypothesized to be associated with the function of BMSCs in the HSC niche, were compared. These results may provide evidence for the pathological study of the HSC niche.

\section{Materials and methods}

Clinical characteristics of patients. In total, 10 AML patients (age, 19-60 years; median age, 44 years; six males and four females) and 13 control patients (age, 19-77 years; median age, 48 years; 10 males and three females) were enrolled from the Department of Hematology, Huashan Hospital (Shanghai, China). AML patients included four cases of M2, one of M3, two of M4, one of M5, one cases of M6 and one case that was unclassified. Control patients included eight cases of phase I/II group A B-cell lymphoma, two of phase I/II group A T-cell lymphoma, one of vasculitis, one of splenic hyperfunction and one of bronchitis. All conditions were confirmed by pathological examination and all patients provided informed consent. The experiments met the ethical standards of human experimentation and were approved by the ethics committee of Huashan Hospital. 
Isolation and culture of human BMSCs. Under sterile conditions, $2.5 \mathrm{ml}$ bone marrow was extracted using a $20 \mathrm{ml}$ syringe from the posterior superior iliac spine of the AML and control patients. Subsequently, the bone marrow was mixed and diluted with $5 \mathrm{ml}$ phosphate-buffered saline (PBS). The cell suspension was added to $3.5 \mathrm{ml}$ Ficoll extraction liquid (Pharmacia, Uppsala, Sweden)and centrifuged at $432 \mathrm{x}$ g for $20 \mathrm{~min}$. The single nucleus layer was transferred to another centrifuge tube, and washed twice with $10 \mathrm{ml}$ Dulbecco's modified Eagle's medium-low glucose (DMEM-LG; Gibco, Grand Island, NY, USA) medium (containing 10\% fetal bovine serum (Gibco) and $100 \mathrm{U} / \mathrm{ml}$ penicillin-streptomycin; Gibco). The cells were cultured at a density of $5 \times 10^{5} / \mathrm{cm}^{2}$ in $5 \% \mathrm{CO}_{2}$ incubator (Heal Force, Hong Kong, SAR, China) at $37^{\circ} \mathrm{C}$. The medium was changed after $48 \mathrm{~h}$ and non-adherent cells were removed gently. Subsequently, the media were changed every 2-3 days. When cells covered $\sim 90 \%$ of the dish the cells were passaged.

Identification of human BMSCs. The P4 human BMSCs were digested for 2 min with $0.25 \%$ Trypsin/EDTA (Gibco), 10 -fold volume of DMEM-LG medium was used to terminate the digestion, the solution was centrifuged at $260 \mathrm{x} \mathrm{g}$ for $5 \mathrm{~min}$ and the supernatant was removed. The cells were washed and resuspended with $0.5 \mathrm{ml}$ cold PBS at a density of $10^{6} / \mathrm{ml}$. Human CD73-peridinin chlorophyl (PerCP, CD105-phycoerythrin (PE), CD90-allophycocyanin (APC), CD45-fluorescein isothiocyanate (FITC) monoclonal antibodies (eBioscience, San Diego, CA, USA) and their isotype controls were added to the cell suspension and incubated for $30 \mathrm{~min}$ on ice, then washed and resuspended with $0.5 \mathrm{ml}$ cold PBS. The samples were detected by flow cytometry and analyzed by Cell Quest software (Beckman Coulter, Brea, CA, USA). Each sample included a minimum of 10,000 cells.

Differentiation of human BMSCs. P4 human BMSCs were digested for 2 min with $0.25 \%$ Trypsin/EDTA, then resuspended by adipose induction medium (containing DMEM-LG medium, $4 \mathrm{mM}$ L-glutamine, $10 \mu \mathrm{g} / \mathrm{ml}$ bovine insulin, $0.25 \mu \mathrm{M}$ dexamethasone, $0.5 \mathrm{mM}$ IBXM, $50 \mu \mathrm{M}$ indomethacin) or osteoblast induction medium (containing DMEM-LG medium, $0.2 \mathrm{mM}$ vitamin $\mathrm{C}, 0.1 \mathrm{uM}$ dexamethasone, $10 \mathrm{mM}$ $\beta$-glycerophosphate), respectively. All buffer components were from Sigma (St. Louis, MO, USA) The cells were seeded in 6 -well plates at a density of $5 \times 10^{4}$ per well. The induction medium was changed every 2-3 days and photographed.

Oil red $O$ staining. Following 14 days of adipose induction, the medium was abandoned and washed twice with PBS. Subsequently the cells were frozen at $-20^{\circ} \mathrm{C}$ for $20 \mathrm{~min}$, the cells were fixed with $4 \%$ paraformaldehyde (PFA) for $20 \mathrm{~min}$ and washed twice with PBS. To this $0.5 \%$ Oil Red O solution (Sigma)was added for $20 \mathrm{~min}$ and the cells were subsequently washed twice with PBS. The cells were stained with hematoxylin for 5 min and washed twice with PBS.

Alizarin red staining. Following 14 days of osteoblast induction, the medium was abandoned and washed twice with PBS. The cells were fixed with $95 \%$ ethanol for $10 \mathrm{~min}$ and washed twice with PBS. Alizarin red (1\%; Sigma) was added to $2 \%$ ethanol for $5 \mathrm{~min}$ and washed twice with PBS.
Quantitative polymerase chain reaction ( $q P C R)$. TRIzol (1 ml; Invitrogen Life Technologies, Carlsbad, CA, USA) was added to $\mathrm{P} 4$ cultured cells at room temperature for $5 \mathrm{~min}$, subsequently $0.2 \mathrm{ml}$ chloroform was added for $3 \mathrm{~min}$. The solution was centrifuged at $12,000 \mathrm{x}$ g for $15 \mathrm{~min}$ and the supernatant was removed. Isopropanol $(0.5 \mathrm{ml})$ was added at room temperature for $10 \mathrm{~min}$, centrifuged at $12,000 \mathrm{x} \mathrm{g}$ for $10 \mathrm{~min}$ and the supernatant was subsequently removed. In order to wash the precipitate, $1 \mathrm{ml}$ $75 \%$ ethanol was added, centrifuged at $7500 \mathrm{x}$ g for $5 \mathrm{~min}$ and the supernatant was subsequently removed and dissolved in RNAase free $\mathrm{H}_{2} \mathrm{O}$. Reverse transcription was performed using an RT-PCR kit (Takara, Otsu, Japan) in accordance with the manufacturers instructions. cDNA $(2 \mu \mathrm{l})$ was obtained and $1 \mu \mathrm{M}$ each of the upstream and downstream primers (Invitrogen Life Technologies), $10 \mu \mathrm{l}$ master mix and $6 \mu \mathrm{l}$ DEPC water were added. Amplification was performed for 40 cycles (GeneAmp PCR system 9700; Applied Biosystems, Foster City, CA,USA) and the data was analyzed using the relative quantification method (UV-2000 spectrophotometer; Unico, Franksville, WI, USA). Primers sequences used are as follows: Sense: 5'-GAGA TTTCTCTGTATGGCACC-3' and antisense: 5'-CTGCAAAT GAGACACTTTCTC-3' for Lipoprotein lipase (LPL); sense: 5'-TTGCAGCCTTCTCAGCCAA-3' and antisense: 5'-GGAG GCAAAAGCAAATCACTG-3' for osteopontin (OPN); sense: 5'-TTCCCTCGACACCCGATTC-3' and antisense: 5'-TAGGTGGAGTCCCAGGCGTA-3' for E-Cadherin; sense: 5'-GGTGGAGGAGAAGAAGAC-3' and antisense: 5'-GGCATCAGGCTCCACAGT-3' for N-Cadherin; and sense: 5'-GGATTTGGTCGTATTGGG-3' and antisense: 5'-GGAAGATGGTGATGGGATT-3' for GAPDH.

Western blotting. P4 cultured cells were lysed in $0.2 \mathrm{ml}$ lysis buffer [0.1\% SDS, 1\% NP-40, 50 mM HEPES, pH 7.4, $2 \mathrm{mM}$ EDTA, $100 \mathrm{mM} \mathrm{NaCl}, 5 \mathrm{mM}$ sodium orthovanadate (all Sigma), $40 \mu \mathrm{M}$ PNPP (Santa Cruz Biotechnology, Inc., Dallas, TX, US) and 1\% Protease Inhibitor Cocktail set I (Calbiochem, La Jolla, CA, USA)]. Lysates were centrifuged at $4,000 \mathrm{x} \mathrm{g}$ for $25 \mathrm{~min}$. The supernatant was obtained and denatured directly for western blotting. Subsequently, samples were separated by electrophoresis in 10\% SDS-PAGE and transferred to a polyvinylidene fluoride membrane. The membranes were blocked for $2 \mathrm{~h}$ at room temperature in $5 \%$ bovine serum albumin, followed by overnight incubation at $4^{\circ} \mathrm{C}$ with the primary antibody, then rinsed and incubated for $1 \mathrm{~h}$ with the peroxidase-conjugated goat anti-rabbit secondary antibody (Sigma). Membranes were then rinsed in Tris-buffered saline with Tween-20 three times and visualized using enhanced chemiluminescence (ECL) (Amersham Pharmacia Biotech, Piscataway, NJ, USA). The primary antibodies used were as follows: Anti-LPL (polyclonal, Santa Cruz Biotechnology Inc., 1:500), anti-OPN (polyclonal, Santa Cruz Biotechnology Inc., 1:500), anti-tubulin (monoclonal, Sigma, 1:5,000).

Statistical analysis. All data were obtained from a minimum of three independent experiments. A non-parametric test was performed between the different groups. $\mathrm{P}<0.05$ was considered to indicate a statistically significant difference. Excel (Microsoft, Redmond, Washington, USA) and SPSS 5.0 software (SPSS Inc., Chicago, IL, USA) was used for the statistical tests. 

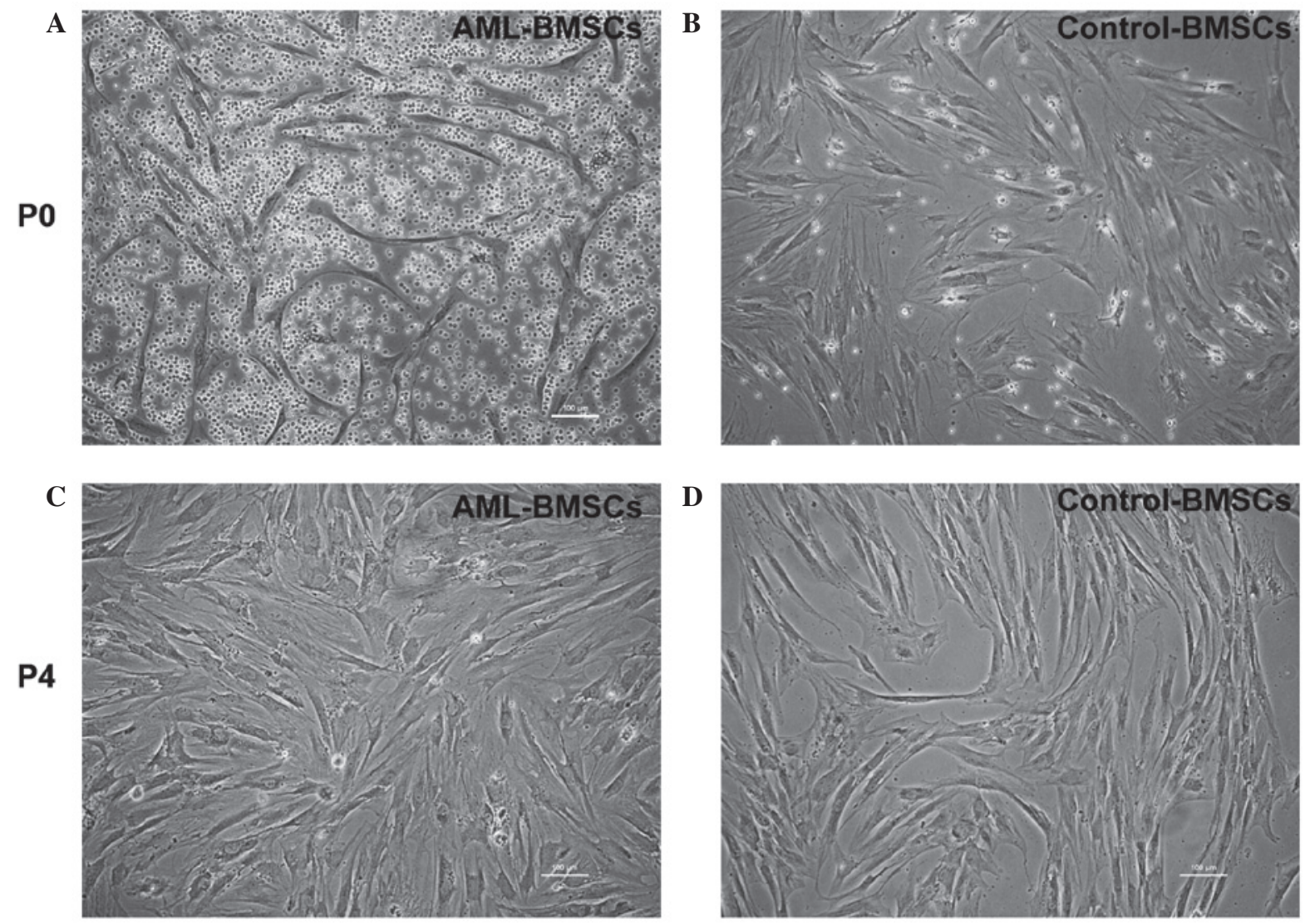

Figure 1. Morphological analyses of bone marrow mesenchymal stem cells (BMSCs). (A and B) Primary BMSCs from acute myeloid leukemia (AML) patients demonstrated an aberrant morphology compared with the controls. (C and D) Following four generations, the two groups of cells presented with a similar spindle morphology. Scale bar, $100 \mu \mathrm{m}$
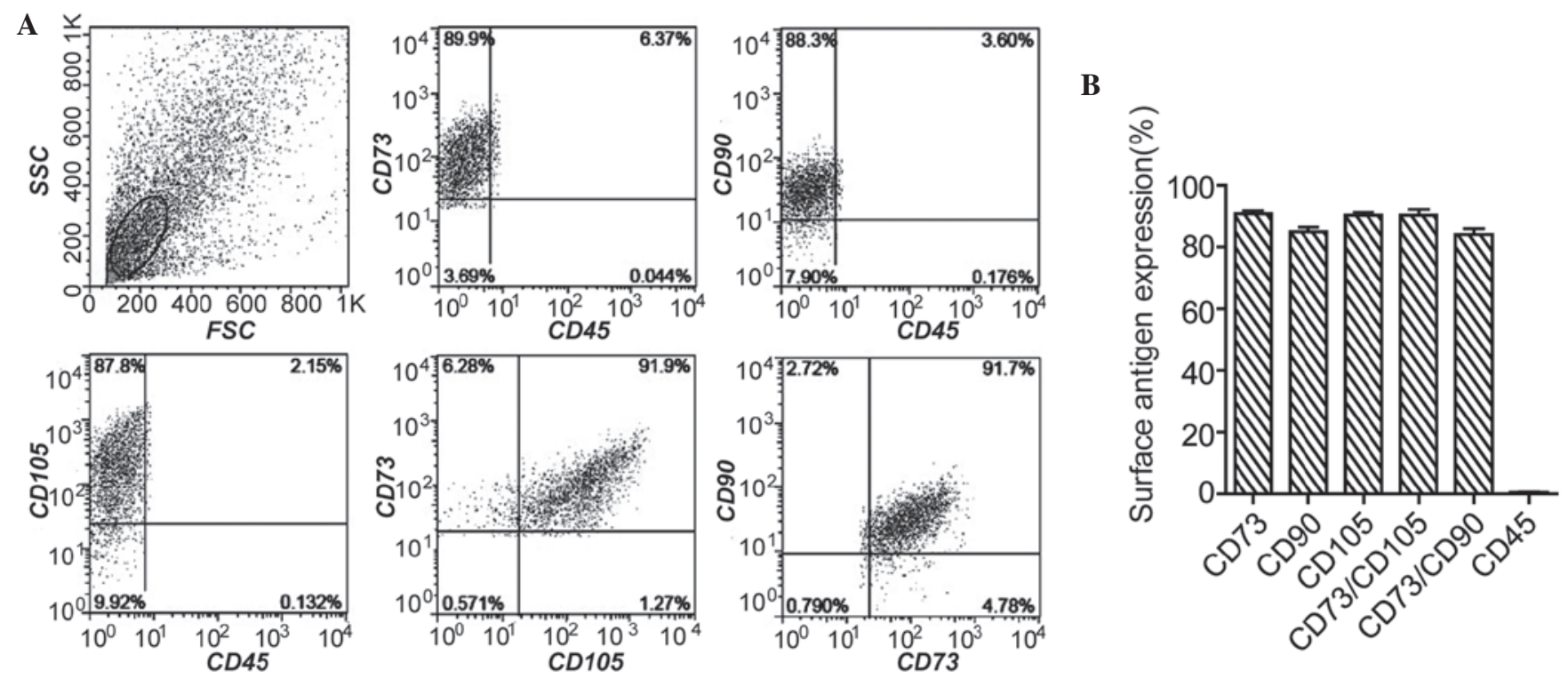

Figure 2. Surface marker identification of bone marrow mesenchymal stem cells (BMSCs). (A and B) Flow cytometry identified BMSC positive marker CD105, CD73, CD90 and negative marker CD45. Bar diagram represents percentages of cells exhibiting marker expression.

\section{Results}

Morphology and phenotype of BMSCs. BMSCs were collected from patients diagnosed with AML $(n=10)$ and controls $(n=13)$. BMSCs derived from the AML patients and controls were cultured in the same culture medium and were capable of being passaged several times.

Normally, primary cultured BMSCs exhibit a spindle-shaped morphology with a few round suspended cells. However, the BMSCs from the AML patients exhibited a more 
A

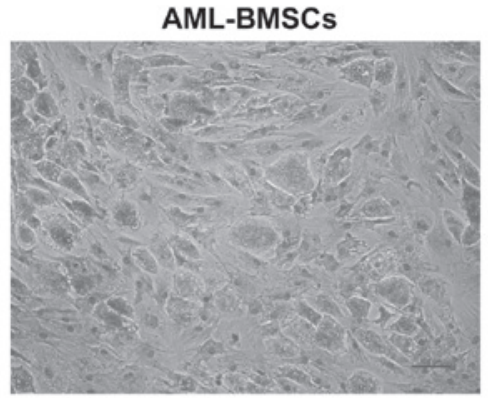

Control-BMSCs

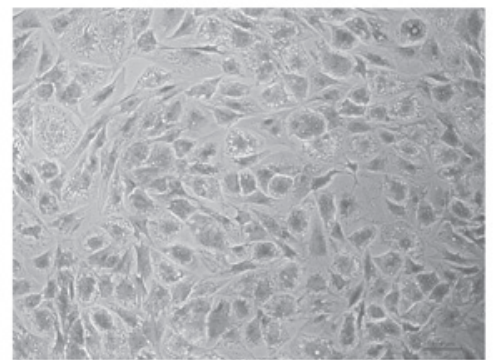

B

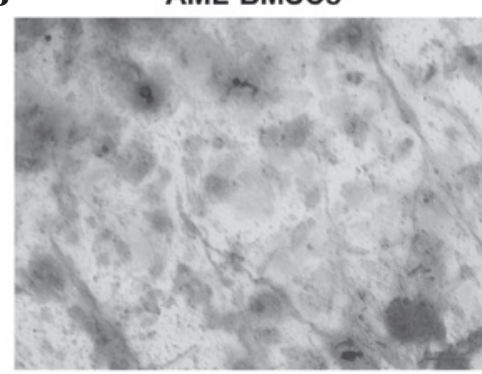

Control-BMSCs

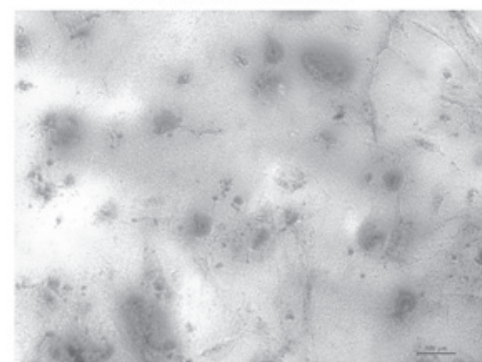

Figure 3. Oil red and alizarin red staining following adipogenic and osteogenic differentiation on day 14 . (A) Day 14 of adipogenic differentiation, oil red staining of bone marrow mesenchymal stem cells (BMSCs) of the patients with acute myeloid leukemia (AML) and the controls. (B) Day 14 of osteogenic differentiation, alizarin red staining of BMSCs of AML patients and controls. Scale bar, $100 \mu \mathrm{m}$

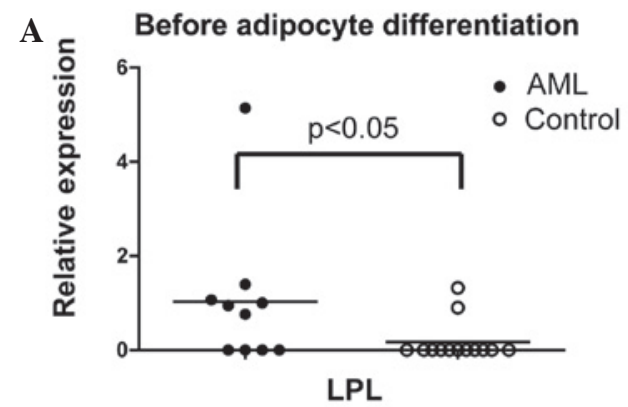

B
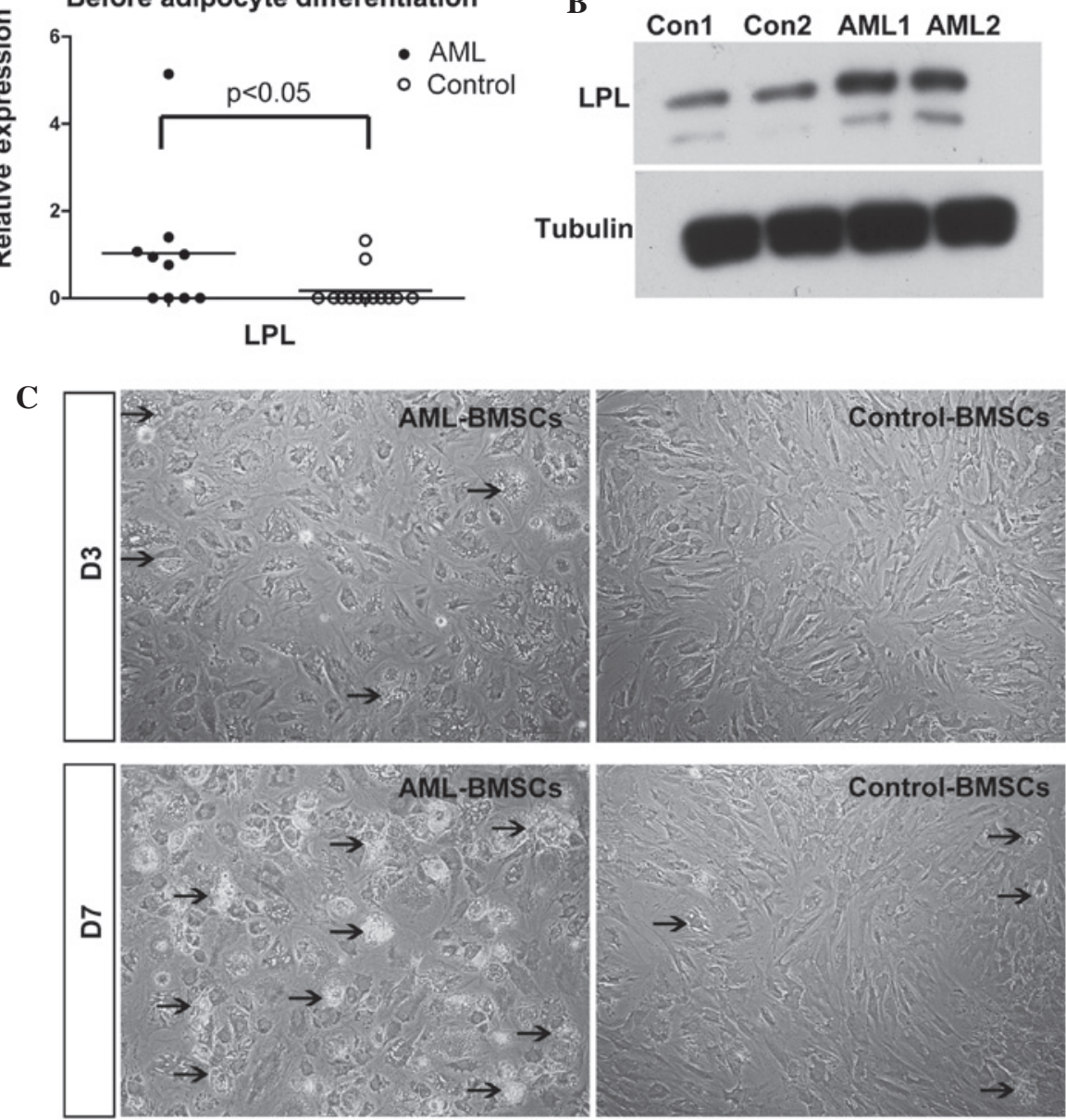

Figure 4. Comparison of adipogenic differentiation ability. (A) Prior to adipogenic differentiation, the mRNA level of lipid marker gene lipoprotein lipase (LPL) from bone marrow mesenchymal stem cells (BMSCs) of patients with acute myeloid leukemia (AML) was significantly higher than controls. (B) Prior to adipogenic differentiation, the protein level of LPL from the BMSCs of patients with AML was significantly higher than those from the controls. (C) Following adipogenic differentiation, lipid drops appeared early on day three and increased in number in the BMSCs of AML patients by day seven, lipid drops are indicated by black arrows. Scale bar, $100 \mu \mathrm{m}$. 
A

Before osteoblast differentiation
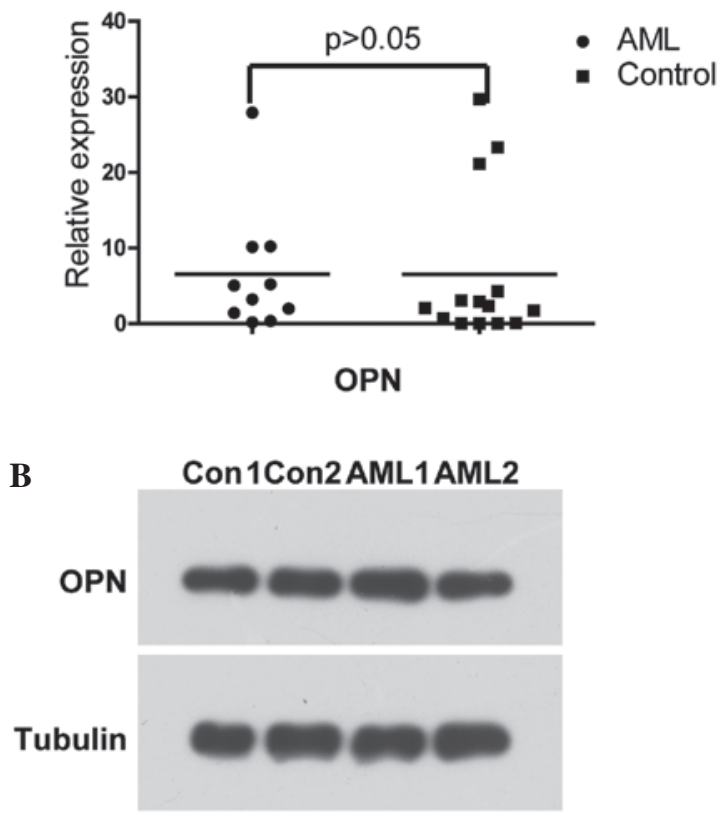

Figure 5. Comparison of osteogenic differentiation ability. (A) Prior to osteogenic differentiation, mRNA level of osteopontin (OPN) from the bone marrow mesenchymal stem cells (BMSCs) of patients with acute myeloid leukemia (AML) exhibited no differences compared with the controls (B) Prior to osteogenic differentiation, the protein level of OPN from the BMSCs of patients with AML exhibited no differences when compared with the controls.

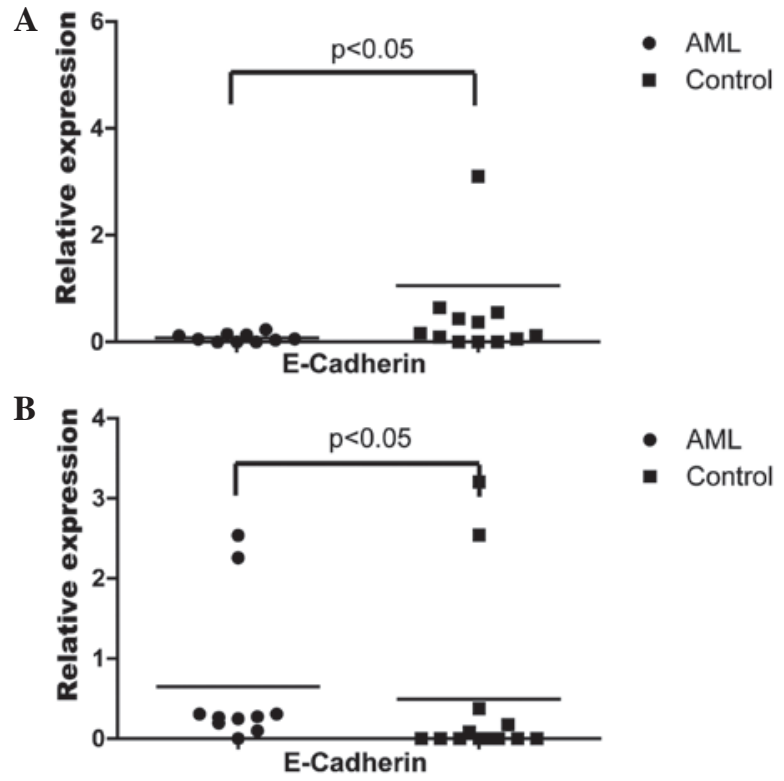

Figure 6. The mRNA level of E-cadherin in BMSCs following adipogenic and osteogenic differentiation. (A) E-cadherin exhibited a significantly lower mRNA level in the bone marrow mesenchymal stem cells (BMSCs) of patients with acute myeloid leukemia (AML) compared with the controls following adipogenic differentiation. (B) Following osteogenic differentiation, E-cadherin demonstrated a significant higher mRNA level in the BMSCs of AML patients compared with the controls.

irregular morphology, with an increased number of branches and a greater number of round cells compared with those from the controls (Fig. 1A and B). Following passaging four times, the two groups of cells presented with a similar normal, long spindle shape. (Fig. 1C and D)

When the results were sorted by flow cytometry, it revealed that these BMSCs expressed positive surface markers CD105, CD73 and CD90, and the negative surface marker, CD45 (Fig. 2A and B). Furthermore, following differentiation, the two groups of BMSCs may differentiate into adipocytes and osteocytes (Fig. 3). Taken together, these results confirmed that these isolated cells were BMSCs.

Comparison of adipogenic and osteogenic differentiation ability. Subsequently, BMSCs from patients with AML and the controls were tested for their differentiation ability. Prior to induction, the qPCR results revealed that the mRNA levels of the lipid cell marker LPL in the AML group were significantly higher than those identified in the controls $(\mathrm{P}<0.05$, Fig. 4A). The results for the protein levels revealed similar results (Fig. 4B). Following three days of adipogenic induction, lipid drops were only detected in the BMSCs of AML patients. Furthermore, there were more lipid droplets in the BMSCs of AML patients than in those of the controls following seven days of adipogenic induction (Fig. 4C). These results demonstrated that the BMSCs of AML patients tended to pre-differentiate to adipocytes.

Prior to osteogenic differentiation, the mRNA and protein levels of OPN exhibited no differences between the two groups (Fig. 5A and B). Furthermore, no differences in the expression of OPN were observed between the two groups following osteogenic differentiation (data not shown).

Following 14 days of adipogenic and osteogenic differentiation, oil red $\mathrm{O}$ staining and alizarin red staining revealed that the BMSCs of AML patients were capable of differentiating into adipocytes and osteocytes. The staining revealed no differences between the BMSCs of the AML patients and those of the controls (Fig. 3).

mRNA levels of $N$ - and E-Cadherin in the BMSCs of AML patients and controls prior to and following differentiation. The BMSCs from AML patients and the controls expressed $\mathrm{N}$-Cadherin/E-Cadherin and their mRNA levels revealed no significant differences prior to differentiation (data not shown). Following adipogenic induction, the mRNA level of E-cadherin in the BMSCs of AML patients was significantly lower than that of the controls following adipogenic induction (Fig. 6A). Following osteogenic induction, the mRNA level of E-cadherin in the BMSCs of the AML patients was significantly higher compared with the controls following osteogenic induction (Fig. 6B). By contrast, N-cadherin was not identified to be differentially expressed between the two groups following differentiation (data not shown).

\section{Discussion}

The bone marrow HSC niche is essential for the maintenance of HSCs. The HSC niche alternation was hypothesized to be the major cause of the malignant myeloid disorder AML. However, little is known with regard to the alteration of BMSCs in AML patients. The results of the present study demonstrated that BMSCs from AML patients exhibited certain differing characteristics compared with the controls. 
Primary cultured BMSCs from AML patients exhibited an irregular morphology compared with those from the controls. In addition, BMSCs from AML patients exhibited higher lipid marker LPL expression prior to adipogenic differentiation and pre-differentiated to adipocytes. Furthermore, following differentiation, the mRNA levels of E-cadherin but not $\mathrm{N}$-cadherin in the BMSCs of AML patients were identified to be significantly different from the controls.

The morphological changes of primary BMSCs may influence the function of BMSCs in the HSC niche as the support cells. This difference disappeared following several generations in an in vitro culture, which suggests that the morphological changes may arise from in vivo pathological conditions.

The results of the present study demonstrated that BMSCs from AML patients were capable of differentiation into osteoblasts and adipocytes following 14 days of in vitro induction. However, BMSCs of AML patients tended to pre-differentiate into adipocytes. While osteocytes were hypothesized to be positive regulators of the HSC niche $(6,7)$, adipocytes were identified as negative regulators of the HSC niche (8). The expansion of adipocytes harmed the maintenance of the HSC niche. Therefore, BMSCs from the AML patients may predifferentiate to adipocytes in vivo, leading to HSC depletion. As the results did not exhibit any difference in the capacity for osteogenic differentiation, the HSC niche alternation in AML patients may not be derived from the increasing or depletion of BMSCs-derived osteocytes.

Cell-cell adhesion mediates cell migration and signaling transduction, which is vital for HSC niche maintenance (9). Several reports have emphasized the function of N-cadherin in the homing and maintenance of HSCs $(10,11)$. However, knocking out $\mathrm{N}$-cadherin in mice did not lead to HSC depletion $(12,13)$. One potential explanation is that another redundant adhesion molecule compensates for the function of $\mathrm{N}$-cadherin. The current results also exhibited no differences in the expression of $\mathrm{N}$-cadherin between BMSCs from AML patients and those from the controls. However, the mRNA from E-caderin was detected in the BMSCs from AML patients and controls and the mRNA levels of E-cadherin in the BMSCs of AML patients were significantly different from the controls following differentiation. As a result, adipocytes and osteocytes derived from BMSCs may have different signaling transduction and motility capabilities.

The mechanism for the alteration of BMSCs may arise from a factor secreted from malignant cells, such as stem cell factor (SCF), tumor necrosis factor (TNF) or macrophage colony stimulating factor (M-CSF) in the bone marrow (14).

In conclusion, it was identified that BMSCs from AML patients exhibited different morphologies, differentiation capability and adhesion molecular expression from the controls in vitro. These results may further the understanding of the HSC niche in pathological conditions.

\section{Acknowledgements}

The authors would like to thank the patients and staff of the Department of Hematology of Huashan Hospital (Shanghai, China) for the donation and collection of bone marrow samples. This study was supported by the National Basic Research Program of China (grant no. 2011CB910404), grants from National Natural Science Foundation of China (grant no. 81070399, 31371480), and Foundation from Science and Technology Commission of Shanghai Municipality (grant no. 13JC1406404) to T.C.

\section{References}

1. Pittenger MF, Mackay AM, Beck SC, et al: Multilineage potential of adult human mesenchymal stem cells. Science 284: 143-147, 1999.

2. Méndez-Ferrer S, Michurina TV, Ferraro F, Mazloom AR, Macarthur BD, Lira SA, Scadden DT, Ma'ayan A, Enikolopov GN and Frenette PS: Mesenchymal and haematopoietic stem cells form a unique bone marrow niche. Nature 466: 829-834, 2010.

3. Wang LD and Wagers AJ: Dynamic niches in the origination and differentiation of haematopoietic stem cells. Nat Rev Mol Cell Biol 12: 643-655, 2011.

4. Kiel MJ and Morrison SJ: Uncertainty in the niches that maintain haematopoietic stem cells. Nat Rev Immunol 8: 290-301, 2008.

5. Steffen B, Müller-Tidow C, Schwäble J, Berdel WE and Serve H: The molecular pathogenesis of acute myeloid leukemia. Crit Rev Oncol Hematol 56: 195-221, 2005.

6. Calvi LM, Adams GB, Weibrecht KW, et al: Osteoblastic cells regulate the haematopoietic stem cell niche. Nature 425: 841-846, 2003.

7. Zhang J, Niu C, Ye L, et al: Identification of the haematopoietic stem cell niche and control of the niche size. Nature 425: 836-841, 2003.

8. Naveiras O, Nardi V, Wenzel PL, Hauschka PV, Fahey F and Daley GQ: Bone-marrow adipocytes as negative regulators of the haematopoietic microenvironment. Nature 460: 259-263, 2009.

9. Takeichi M: Cadherins: A molecular family important in selective cell-cell adhesion. Annu Rev Biochem 59: 237-252, 1990.

10. Hosokawa K, Arai F, Yoshihara H, et al: Cadherin based adhesion is a potential target for niche manipulation to protect hematopoietic stem cells in adult bone marrow. Cell Stem Cell 6: 194-198, 2010.

11. Hosokawa K, Arai F, Yoshihara H, etal: Knockdown of N-cadherin suppresses the long-term engraftment of hematopoietic stem cells. Blood 116: 554-563, 2010.

12. Kiel MJ, Radice GL and Morrison SJ: Lack of evidence that hematopoietic stem cells depend on N-cadherin-mediated adhesion to osteoblasts for their maintenance. Cell Stem Cell 1: 204-217, 2007.

13. Kiel MJ, Acar M, Radice GL and Morrison SJ: Hematopoietic stem cells do not depend on N-cadherin to regulate their maintenance. Cell Stem Cell 4: 170-179, 2009.

14. Wetzler M, Kurzrock R, Estrov Z, Estey E and Talpaz M: Cytokine expression in adherent layers from patients with myelodysplastic syndrome and acute myelogenous leukemia. Leukemia Res 19: 23-34, 1995. 